\title{
UN MODELO DE AUDITORÍA DE CALIDAD AL SERVICIO CONTABLE
}

\author{
A BLUEPRINT TO AUDITING ACCOUNTANT SERVICES QUALITY \\ Román Arturo Valdivia Ramos* \\ Docente Asociado de la Facultad de Ciencias Contables \\ Universidad Nacional Mayor de San Marcos-UNMSM / Lima-Perú \\ [Recepción: Marzo de 2014/ Conformidad: Mayo 2014]
}

\section{RESUMEN}

La pregunta que hace algún tiempo me hice fue...... ¿Quién audita el servicio contable o al personal contable? Esto ha sido el detonante para elaborar este trabajo de Investigación. El contador, el técnico contable, el asistente contable, el auxiliar contable $y$, tantos otros, que laboran en un departamento de contabilidad, creemos que realizan correctamente su trabajo, pues tienen reglas y procedimientos muy claros y precisos a seguir. Sin embargo, quién le hace un seguimiento al personal contable respecto a su trabajo, para saber o conocer, si verdaderamente están cumpliendo con dichas reglas, con los procesos, y procedimientos, con la detección de fallas o errores, con la retroalimentación, con el análisis de la diferencia, así como, normatividad contable, entre otros.

Creo, sinceramente que son pocas las entidades, empresas e instituciones que llevan a cabo un proceso de auditoría de calidad del servicio contable.

Al personal contable, sea contador o asistente, se le evalúa por sus resultados que se plasman en los reportes, EE.FF., y otros documentos que son necesarios para la toma de decisiones en cualquier organización.

Pero, acaso ¿El personal contable no está exento de cometer errores, fallas, deslices u otros? ¡Desde luego que sí!

\section{Palabras Clave:}

Modelo; auditoría; calidad; servicio; contabilidad.

\begin{abstract}
The question I asked myself was some time ago ...... "Who audits the accounting service or accounting staff?". This has been the trigger to make this research work. The accountant, accountant technician, bookkeeper, accounting assistant and many others working in an accounting department, we believe that doing their job properly, they have regulations and very clear and precise procedures to follow. But who makes you follow the accounting staff about their work, to know or meet, if truly compliance with the rules, the processes, procedures, and troubleshooting errors with feedback, the analysis difference, as well as accounting standards, among others.

Sincerely believe that few organizations, companies and institutions that carry out an audit of quality accounting service.

Accounting staff, bookkeeper or assistant, is evaluated by its results which are reflected in the reports, EE.FF., and other documents that are needed for decision-making in any organization.

But was perhaps the accounting staff is not free of errors, faults, slips or others? Certainly you do!
\end{abstract}

\section{Keywords:}

Model; audit; quality; service; accounting.

* Licenciado en Administración. Docente investigador. Email: arturo.valdivia.ramos@gmail.com 


\section{INTRODUCCIÓN}

La auditoría de calidad al servicio contable, es un instrumento que primero permite detectar y luego, eliminar fallas, errores u otros o, por lo menos disminuirlos o corregirlos, para que en otra oportunidad no ocurran tales hechos. Ahí, está el mejoramiento continuo que preconiza la auditoria de calidad para la contabilidad, que es una ciencia y una técnica tan dinámica y cambiante que cada vez, tiene que adecuarse a las características y necesidades de los clientes (sea su propia empresa o un cliente externo). Entonces, ningún servicio contable está exento de mejorarse, siendo uno de los instrumentos importantes (por no decir, el más importante) que hoy en día se tiene, la Auditoría de Calidad.

\section{CARACTERÍSTICAS DE LA AUDITORÍA DE CALIDAD AL SERVICIO CONTABLE}

El trabajo de investigación está compuesto de:

A. Hoja de trabajo de auditoría y cuestionario de auditoría de calidad del servicio contable.

B. Hoja de puntuación de la auditoría de calidad del servicio contable.

C. Informe de auditoría de calidad del servicio contable.

D. Solicitud de acciones correctivas.

\section{A. HOJA DE TRABAJO DE AUDITORÍA}

\begin{tabular}{|c|c|c|c|c|}
\hline \multicolumn{5}{|c|}{ DATOS DE LA EMPRESA } \\
\hline \multicolumn{2}{|c|}{$\begin{array}{l}\text { Nombre: } \\
\text { Distrito: : }-------------------- \\
\text { Teléfono: : }------------------\end{array}$} & \multicolumn{3}{|c|}{$\begin{array}{l}\text { Dirección: : }------------------- \\
\text { Provincia: : }------------------- \\
\text { Página web: :- }-----------------\end{array}$} \\
\hline \multicolumn{5}{|c|}{ CRITERIOS DE AUDITORÍA } \\
\hline \multicolumn{2}{|c|}{$\begin{array}{l}\text { Auditor: }------------------- \\
\text { Auditado: Departamento de Contabilidad }\end{array}$} & \multicolumn{3}{|c|}{$\begin{array}{l}\text { Fecha: .............................. } \\
\text { Ubicación: Gerencia de Contabilidad }\end{array}$} \\
\hline $\mathbf{N}^{\circ}$ & \multicolumn{2}{|c|}{ ELEMENTOS } & $\begin{array}{l}\text { Puntaje } \\
\text { Aprob. } \\
\text { Mínimo }\end{array}$ & $\begin{array}{l}\text { Puntaje } \\
\text { Aprob. } \\
\text { Máximo }\end{array}$ \\
\hline 1.0 & \multicolumn{2}{|c|}{$\begin{array}{l}\text { FUNDAMENTOS DE LA CALIDAD APLICADA A LA FUNCIÓN } \\
\text { CONTABLE. }\end{array}$} & & \\
\hline 1.1 & \multirow{2}{*}{\multicolumn{2}{|c|}{$\begin{array}{l}\text { Importancia del sistema de calidad del servicio contable. } \\
\text { Características del sistema de calidad del servicio contable. }\end{array}$}} & 49 & 70 \\
\hline 1.2 & & & 21 & 30 \\
\hline 2.0 & \multicolumn{2}{|c|}{$\begin{array}{l}\text { CARACTERÍSTICAS DE LA CALIDAD OPERATIVA APLICADO A } \\
\text { LA FUNCIÓN CONTABLE }\end{array}$} & & \\
\hline 2.1 & \multicolumn{2}{|c|}{ Características de la calidad contable. } & 98 & 140 \\
\hline 2.2 & \multicolumn{2}{|c|}{ Control de las características de la calidad contable. } & 42 & 60 \\
\hline 3.0 & \multicolumn{2}{|c|}{ PRINCIPIOS DEL SISTEMA DE CALIDAD CONTABLE } & & \\
\hline 3.1 & \multicolumn{2}{|c|}{ Aspectos fundamentales del sistema de calidad en la contabilidad. } & 21 & 30 \\
\hline 3.2 & \multicolumn{2}{|c|}{ Responsabilidad ejecutiva de la contabilidad. } & 21 & 30 \\
\hline 3.3 & \multicolumn{2}{|l|}{ Desarrollo y apoyo al personal contable. } & 28 & 40 \\
\hline 3.4 & \multicolumn{2}{|c|}{ Estructura del sistema de la calidad contable. } & 21 & 30 \\
\hline 3.4 .1 & \multicolumn{2}{|c|}{ Documentación del sistema de calidad contable. } & 21 & 30 \\
\hline 3.4 .2 & \multicolumn{2}{|c|}{ Normas del sistema de la calidad contable. } & 21 & 30 \\
\hline 3.4 .3 & \multicolumn{2}{|c|}{ Análisis de diferencias en el desempeño contable. } & 21 & 30 \\
\hline 3.4 .4 & \multicolumn{2}{|c|}{ Informe de auditoría de calidad del servicio contable. } & 28 & 40 \\
\hline 3.4 .5 & \multicolumn{2}{|c|}{ Vigilancia de las interacciones con los clientes. } & 28 & 40 \\
\hline
\end{tabular}

76/ QUIPURAMAYOC | Vol. 22(41) 2014 


\begin{tabular}{|c|l|c|c|}
\hline 4.0 & OPERACIONES DEL SISTEMA DE LA CALIDAD CONTABLE & & \\
4.1 & Control del proceso contable. & 14 & 20 \\
4.1 .1 & Definición y objetivos. & 21 & 30 \\
4.1 .2 & Relaciones con los clientes. & 7 & 10 \\
4.1 .3 & Proyectos contables de mejora. & 14 & 20 \\
4.1 .4 & Nuevas propuestas contables. & 7 & 10 \\
4.1 .5 & Registros contables normalizados y/o estandarizados. & 14 & 20 \\
4.1 .6 & Administración contable. & 21 & 30 \\
4.1 .7 & Control del proceso administrativo (P-O-C-D-C). & 7 & 10 \\
4.1 .8 & Revisión de la documentación contable. & & \\
4.2 & Control del proceso de rediseño. & 14 & 20 \\
4.2 .1 & Historia del rediseño. & 74 & 20 \\
4.2 .2 & Identificar actividades básicas del personal contable. & 7 & 10 \\
4.2 .3 & Detectar problemas en los procesos actuales del trabajo contable. & 7 & 10 \\
4.2 .4 & Desarrollar nuevos procesos y procedimientos. & 7 & 10 \\
4.2 .5 & Desarrollar nuevas mediciones de desempeño del personal contable. & 7 & 10 \\
4.2 .6 & Especificar las nuevas normas del desempeño del personal contable. & & \\
4.2 .7 & Evaluar los nuevos procesos y procedimientos del trabajo contable. & 14 & 20 \\
4.3 & Evaluación del proceso del servicio al cliente. & 14 & 20 \\
4.3 .1 & Profesionalismo del personal contable en general. & 14 & 20 \\
4.3 .2 & Retroalimentación del personal contable. & 14 & 20 \\
4.3 .3 & Retroalimentación de satisfacción de los clientes. & 7 & \\
4.3 .4 & Control del sistema de medición. & 21 & 30 \\
4.4 & Análisis de diferencias en el desempeño y la mejora contínua. & 28 & 40 \\
4.4 .1 & Ejecución y uso. & 700 & 1000 \\
4.4 .2 & Sistema de recolección de datos. & & \\
4.4 .3 & Iniciativa de mejora de calidad del servicio contable. & & \\
\hline TOTAL & PUNTAJE DE AUDITORÍA & & \\
\hline
\end{tabular}

Fuente: Propia. 


\begin{tabular}{|c|c|c|c|c|c|c|c|c|c|c|}
\hline \multicolumn{11}{|c|}{ CUESTIONARIO DE AUDITORÍA DE CALIDAD DEL SERVICIO CONTABLE } \\
\hline \multicolumn{2}{|c|}{ Auditado: } & \multicolumn{9}{|l|}{ Auditor: } \\
\hline \multicolumn{2}{|c|}{ Gerencia: } & \multicolumn{9}{|l|}{ Número de Auditoría: } \\
\hline \multicolumn{2}{|c|}{ Fecha de la Auditoría: } & \multicolumn{9}{|l|}{ Referencia: } \\
\hline \multirow{3}{*}{$\begin{array}{l}\mathrm{N}^{\circ} \mathrm{DE} \\
\mathrm{REF}\end{array}$} & \multirow{3}{*}{\multicolumn{2}{|c|}{ PREGUNTA }} & \multirow{3}{*}{$\mathrm{N}^{\circ}$} & \multicolumn{6}{|c|}{ PUNTAJE } & \multirow{3}{*}{$\begin{array}{l}\text { NOTAS } \\
\text { DEL } \\
\text { AUDI- } \\
\text { TOR }\end{array}$} \\
\hline & & & & \multicolumn{3}{|c|}{$\begin{array}{c}\text { NO } \\
\text { CUMPLE }\end{array}$} & \multicolumn{3}{|c|}{$\begin{array}{c}\text { SI } \\
\text { CUMPLE }\end{array}$} & \\
\hline & & & & 0 & 2 & 4 & 6 & 8 & 10 & \\
\hline \multirow[t]{4}{*}{1.0} & $\begin{array}{l}\text { Fundamento de la calidad } \\
\text { ¿Se ha definido la importancia que tiene la satisfacción d }\end{array}$ & e los clientes? & 1 & & & & & & & \\
\hline & ¿Se ha definido la importancia que tiene el capital human & no contable? & 2 & & & & & & & \\
\hline & ¿Se ha definido las características de calidad de servicio c & ontable? & 3 & & & & & & & \\
\hline & ¿Se ha definido las características de competitividad del $\mathrm{t}$ & rabajador contable? & 4 & & & & & & & \\
\hline \multirow[t]{7}{*}{2.0} & $\begin{array}{l}\text { Características de la calidad operativa } \\
\text { ¿Se ha definido las características de productividad del se }\end{array}$ & ervicio contable? & 5 & & & & & & & \\
\hline & ¿Se ha definido las normas de aceptabilidad para el servic & cio contable? & 6 & & & & & & & \\
\hline & ¿Se ha definido las normas de la eficiencia contable? & & 7 & & & & & & & \\
\hline & ¿Se ha definido las normas de la eficacia contable? & & 8 & & & & & & & \\
\hline & ¿La gerencia de contabilidad tiene la responsabilidad de & medir la calidad? & 9 & & & & & & & \\
\hline & ¿La gerencia de contabilidad verifica datos y resultados & de la medición? & 10 & & & & & & & \\
\hline & ¿La gerencia de Contabilidad toma acciones en base a los & resultados obtenidos? & 11 & & & & & & & \\
\hline \multirow[t]{4}{*}{$\begin{array}{c}3.0 \\
3.1\end{array}$} & $\begin{array}{l}\text { Principios del sistema de calidad contable } \\
\text { ¿La gerencia de contabilidad define la calidad contable se } \\
\text { tes? }\end{array}$ & egún establecen los clien- & 12 & & & & & & & \\
\hline & ¿La gerencia de contabilidad define la calidad contable d & e la empresa? & 13 & & & & & & & \\
\hline & ¿La gerencia de contabilidad define la calidad contable $\mathrm{d}$ & el proceso operativo? & 14 & & & & & & & \\
\hline & ¿La gerencia de contabilidad define la calidad contable $\mathrm{q}$ & ue debe tener el personal? & 15 & & & & & & & \\
\hline \multirow[t]{3}{*}{3.2} & ¿La gerencia de contabilidad ha desarrollado un manual & de calidad? & 16 & & & & & & & \\
\hline & ¿La gerencia de contabilidad autoriza la ejecución de aud & litorías de calidad? & 17 & & & & & & & \\
\hline & ¿La gerencia de contabilidad revisa y/o verifica de forma & continua los resultados? & 18 & & & & & & & \\
\hline \multirow[t]{6}{*}{3.3} & $\begin{array}{l}\text { ¿La gerencia de contabilidad reconoce y recompensa las } \\
\text { nal? }\end{array}$ & contribuciones del perso- & 19 & & & & & & & \\
\hline & ¿La gerencia de contabilidad proporciona la instrucción & necesaria al personal? & 20 & & & & & & & \\
\hline & ¿La gerencia de contabilidad evalúa las necesidades de in & strucción del personal? & 21 & & & & & & & \\
\hline & ¿La gerencia de contabilidad proporciona la capacitación & necesaria al personal & 22 & & & & & & & \\
\hline & $\begin{array}{l}\text { ¿La gerencia de contabilidad comunica los datos vitales y } \\
\text { sarios? }\end{array}$ & asientos contables nece- & 23 & & & & & & & \\
\hline & ¿El sistema de información existente ayuda de manera ef & icaz al personal? & 24 & & & & & & & \\
\hline \multirow[t]{2}{*}{3.4} & $\begin{array}{l}\text { ¿Existe un sistema para detectar defectos en el sistema op } \\
\text { contable? }\end{array}$ & gerativo del servicio & 25 & & & & & & & \\
\hline & $\begin{array}{l}\text { ¿Existe un sistema, o proceso para corregir los defectos e } \\
\text { contable? }\end{array}$ & el el sistema operativo & 26 & & & & & & & \\
\hline \multirow[t]{3}{*}{3.4 .1} & ¿Están documentadas las expectativas del cliente? & & 27 & & & & & & & \\
\hline & ¿Están documentados los objetivos y las metas de la gere & ncia contable? & 28 & & & & & & & \\
\hline & ¿Están documentados los procedimientos del trabajo cor & htable? & 29 & & & & & & & \\
\hline
\end{tabular}




\begin{tabular}{|c|c|c|c|c|c|c|c|}
\hline & ¿Están documentados el resultado del desempeño contable? & 30 & & & & & \\
\hline & ¿Están documentados la auditoria de la calidad contable? & 31 & & & & & \\
\hline & ¿Están documentadas las actividades correctivas que se llevan a cabo? & 32 & & & & & \\
\hline \multirow[t]{4}{*}{3.4 .2} & ¿Se utilizan normas del desempeño para evaluar el proceso contable? & 33 & & & & & \\
\hline & ¿Se utilizan normas de desempeño para evaluar el proceso administrativo? & 34 & & & & & \\
\hline & ¿Hay normas para el proceso del servicio contable? & 35 & & & & & \\
\hline & ¿Hay normas para el rediseño del proceso contable? & 36 & & & & & \\
\hline \multirow[t]{3}{*}{3.4 .3} & ¿Existen retroalimentación por parte del personal del servicio contable? & 37 & & & & & \\
\hline & ¿Existe retroalimentación por parte de los clientes del servicio contable? & 38 & & & & & \\
\hline & ¿Existe retroalimentación por parte de los auditores de calidad? & 39 & & & & & \\
\hline \multirow[t]{6}{*}{3.4 .4} & ¿El informe de auditoría de calidad, incluye los propósitos y fines? & 40 & & & & & \\
\hline & ¿El informe de auditoría de calidad, incluye los objetivos y operaciones? & 41 & & & & & \\
\hline & ¿El informe de auditoría de calidad, incluye normas de calidad internacional? & 42 & & & & & \\
\hline & ¿El informe de auditoría de calidad, incluye métodos análisis utilizados? & 43 & & & & & \\
\hline & ¿El informe de auditoría de calidad, incluye resumen del informe? & 44 & & & & & \\
\hline & ¿El informe de auditoría de calidad, incluye fechas de seguimiento de acción? & 45 & & & & & \\
\hline \multirow[t]{6}{*}{3.4 .5} & $\begin{array}{l}\text { ¿La gerencia de contabilidad verifica de manera permanente la oportunidad de } \\
\text { entrega? }\end{array}$ & 46 & & & & & \\
\hline & ¿La gerencia de contabilidad revisa constantemente la competitividad del servicio? & 47 & & & & & \\
\hline & ¿La gerencia de contabilidad oculta en el personal las necesidades de información? & 48 & & & & & \\
\hline & $\begin{array}{l}\text { ¿La gerencia de contabilidad de forma periódica verifica la productividad del } \\
\text { personal? }\end{array}$ & 49 & & & & & \\
\hline & ¿La gerencia de contabilidad evalúa la eficiencia de personal contable? & 50 & & & & & \\
\hline & ¿La gerencia de contabilidad evalúa la eficacia del servicio contable? & 51 & & & & & \\
\hline $\begin{array}{l}4.0 \\
4.1\end{array}$ & $\begin{array}{l}\text { Operaciones del Sistema de Calidad Contable } \\
\text { Control del proceso contable. }\end{array}$ & 52 & & & & & \\
\hline \multirow[t]{2}{*}{ 4.1.1 } & ¿Está definido el proceso contable? & 52 & & & & & \\
\hline & $\begin{array}{l}\text { ¿Se utiliza la información contable para establecer objetivos entre el servicio y el } \\
\text { cliente? }\end{array}$ & 53 & & & & & \\
\hline \multirow[t]{4}{*}{4.1 .2} & $\begin{array}{l}\text { ¿El personal contable documenta la información para determinar las metas del } \\
\text { cliente? }\end{array}$ & 54 & & & & & \\
\hline & ¿El personal documenta las estrategias para ayudar a los clientes a tener más éxito? & 55 & & & & & \\
\hline & ¿El personal contable documenta los problemas del cliente? & 56 & & & & & \\
\hline & ¿El personal contable documenta las fortalezas y debilidades de la competencia? & 57 & & & & & \\
\hline 4.1 .3 & $\begin{array}{l}\text { ¿El personal contable participa en las actividades de proyectos de mejora de } \\
\text { procesos? }\end{array}$ & 58 & & & & & \\
\hline \multirow[t]{2}{*}{4.1 .4} & $\begin{array}{l}\text { ¿Existe un procedimiento para seguir las propuestas planteadas por el servicio } \\
\text { contable? }\end{array}$ & 59 & & & & & \\
\hline & ¿Existe un procedimiento para desarrollar las propuestas contables? & 60 & & & & & \\
\hline 4.1 .5 & ¿Existe un procedimiento para colocar las cuentas? & 61 & & & & & \\
\hline \multirow[t]{2}{*}{4.1 .6} & ¿La gerencia de contabilidad dirige acciones para mejorar las prácticas contables? & 62 & & & & & \\
\hline & ¿Se informan los resultados de las acciones contables? & 63 & & & & & \\
\hline \multirow[t]{2}{*}{ 4.1.7 } & ¿Se encuentran documentados los procedimientos contables? & 64 & & & & & \\
\hline & $\begin{array}{l}\text { ¿Los procedimientos documentados se revisan para evaluar su eficiencia - efica- } \\
\text { cia? }\end{array}$ & 65 & & & & & \\
\hline
\end{tabular}




\begin{tabular}{|c|c|c|c|c|c|c|c|}
\hline & $\begin{array}{l}\text { ¿Se revisan las prácticas contables para cumplir con los procedimientos aproba- } \\
\text { dos? }\end{array}$ & 66 & & & & & \\
\hline & ¿Se asigna tiempo a las actividades contables? & 67 & & & & & \\
\hline 4.1 .8 & ¿Se detecta y elimina los procesos contables innecesarios? & 68 & & & & & \\
\hline \multirow[t]{3}{*}{$\begin{array}{l}4.2 \\
4.2 .1\end{array}$} & $\begin{array}{l}\text { Control del proceso de rediseño. } \\
\text { ¿Se he rediseñado el sistema de calidad del servicio en los últimos años? }\end{array}$ & 69 & & & & & \\
\hline & ¿Se ha rediseñado el proceso administrativo durante los últimos años? & 70 & & & & & \\
\hline & ¿Se ha rediseñado los procedimientos durante los últimos años? & 71 & & & & & \\
\hline \multirow[t]{2}{*}{4.2 .2} & ¿Se ha identificado las actividades fundamentales del servicio contable? & 72 & & & & & \\
\hline & $\begin{array}{l}\text { ¿Se ha identificado las oportunidades para mejorar las actividades del servicio } \\
\text { contable? }\end{array}$ & 73 & & & & & \\
\hline 4.2 .3 & $\begin{array}{l}\text { ¿Se identifican y corrigen las actividades que no cumplen con las expectativas del } \\
\text { cliente? }\end{array}$ & 74 & & & & & \\
\hline 4.2 .4 & $\begin{array}{l}\text { ¿Se redefinen los procedimientos para eliminar la causa de insatisfacción de los } \\
\text { clientes? }\end{array}$ & 75 & & & & & \\
\hline 4.2 .5 & $\begin{array}{l}\text { ¿Se desarrollan nuevas mediciones para el trabajo contable que se enfocan al } \\
\text { cliente? }\end{array}$ & 76 & & & & & \\
\hline 4.2 .6 & $\begin{array}{l}\text { ¿Se han desarrollado y utilizado nuevas normas de trabajo que se orienten al } \\
\text { cliente? }\end{array}$ & 77 & & & & & \\
\hline 4.2 .7 & ¿Se aprueban los nuevos procedimientos para la satisfacción de los clientes? & 78 & & & & & \\
\hline \multirow[t]{2}{*}{$\begin{array}{l}4.3 \\
4.3 .1\end{array}$} & $\begin{array}{l}\text { Control del proceso de servicio al cliente. } \\
\text { ¿El personal hace seguimiento a las operaciones para la satisfacción del cliente? }\end{array}$ & 79 & & & & & \\
\hline & ¿El personal sigue los procedimientos de trabajos establecidos? & 80 & & & & & \\
\hline \multirow[t]{2}{*}{4.3 .2} & ¿Se utiliza algún instrumento de retroalimentación para mejorar el trabajo? & 81 & & & & & \\
\hline & ¿El instrumento de retroalimentación detecta obstáculos que enfrenta el personal? & 82 & & & & & \\
\hline \multirow[t]{2}{*}{4.3 .3} & ¿Se mide la performance del personal contable desde la perspectiva del cliente? & 83 & & & & & \\
\hline & $\begin{array}{l}\text { ¿La frecuencia en la medición de la satisfacción de los clientes permite detectar los } \\
\text { puntos de insatisfacción antes de que ocurra un daño o perjuicio a mediano plazo } \\
\text { a la imagen? }\end{array}$ & 84 & & & & & \\
\hline \multirow[t]{3}{*}{ 4.3.4 } & ¿Los cuestionarios, papeles y documentos de trabajo están libres de errores? & 85 & & & & & \\
\hline & $\begin{array}{l}\text { ¿Se lleva a cabo una prueba piloto de la aplicación y/o uso de los documentos de } \\
\text { trabajo? }\end{array}$ & 86 & & & & & \\
\hline & ¿Se comprueba la confiabilidad y validez de los documentos de trabajo? & 87 & & & & & \\
\hline \multirow[t]{2}{*}{$\begin{array}{l}4.4 \\
4.4 .1\end{array}$} & $\begin{array}{l}\text { Análisis de diferencias en el desempeño y la mejora contínua. } \\
\text { ¿Existen diferencias entre el servicio contable y las expectativas de los clientes? }\end{array}$ & 88 & & & & & \\
\hline & $\begin{array}{l}\text { ¿La recolección de datos recaba evidencias para identificar las causas de los defec- } \\
\text { tos? }\end{array}$ & 89 & & & & & \\
\hline \multirow[t]{3}{*}{4.4 .2} & ¿El sistema de recolección de datos capta retroalimentación de los clientes? & 90 & & & & & \\
\hline & ¿El sistema de recolección de datos capta retroalimentación del personal contable? & 91 & & & & & \\
\hline & $\begin{array}{l}\text { ¿El sistema de recolección de datos capta retroalimentación de los auditores de } \\
\text { calidad? }\end{array}$ & 92 & & & & & \\
\hline \multirow[t]{5}{*}{4.4 .3} & $\begin{array}{l}\text { ¿Las iniciativas de mejora de calidad del servicio captan las expectativas del clien- } \\
\text { te? }\end{array}$ & 93 & & & & & \\
\hline & ¿Hay criterios de evaluación del desempeño del personal contable? & 94 & & & & & \\
\hline & ¿Hay oportunidades para reducir costos? & 95 & & & & & \\
\hline & $\begin{array}{l}\text { ¿Hay oportunidades para descubrir los costos ocultos y las actividades no produc- } \\
\text { tivas? }\end{array}$ & 96 & & & & & \\
\hline & ¿Se aceptan la sugerencia de mejora que aportan los clientes? & 97 & & & & & \\
\hline
\end{tabular}




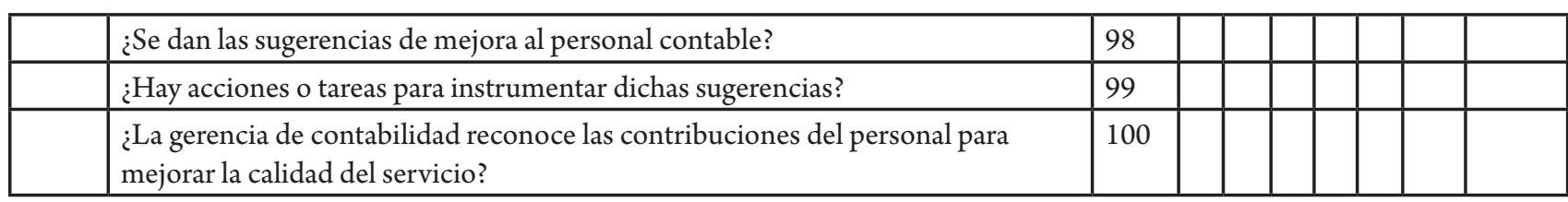

\section{B. HOJA DE PUNTUACIÓN DE LA AUDITORÍA DE CALIDAD DEL SERVICIO CONTABLE}

Ahora se registran los totales en el denominado Cuestionario de Auditoría, que incluye:

- N.o de referencia.

- Puntos de verificación de la auditoría.

- Puntaje mínimo.

- Notas del auditor.

\section{INFORME DE AUDITORÍA DE CALIDAD DEL SERVICIO CONTABLE}

Los informes escritos son fundamentales para toda auditoría. Los informes de auditoría señalan la oportunidad de mejora al auditado, asimismo que dichos informes sirvan para auditorías futuras. La auditoría de calidad del servicio contable termina con la presentación que hace el auditor de las observaciones y hallazgos (hechos encontrados) en el auditado (s).

\section{SOLICITUD DE ACCIONES CORRECTIVAS}

Antes de empezar el proceso de auditoría, la dirección deberá comprender que ésta concluirá con el establecimiento de un programa consistente que asegura la aplicación de las acciones correctivas recomendadas. Todas las desviaciones importantes deberán imponerse (no informar o recomendar) a la dirección en una Hoja Solicitud de Acción Correctiva.

La solicitud de acción correctiva, tal vez sea el elemento más importante de un informe de auditoría de calidad del servicio contable. Las acciones correctivas se jerarquizan de acuerdo a su porcentaje de conformidad con los lineamientos. Sin una acción correctiva, no podrá llevarse la mejora del desempeño, performance o rendimiento por parte de la dirección.

\section{EJEMPLO DE INFORME DE AUDITORÍA}

A continuación, se presenta este ejemplo que ayudará al participante o lector a comprender cómo se lleva a cabo un informe de auditoría de calidad. Observe que el cuestionario es la fuente de la hoja de trabajo de puntuación de la misma, solo se muestra una parte de la citada hoja, seguido de un resumen del total de la puntuación. 


\begin{tabular}{|c|c|c|c|c|c|c|c|}
\hline \multicolumn{8}{|c|}{ CUESTIONARIO DE LA AUDITORÍA } \\
\hline \multirow[b]{2}{*}{$\mathrm{N}^{\circ}$ de referencia de lineamiento de calidad } & \multirow{2}{*}{$\begin{array}{c}\mathrm{N}^{\circ} \text { de pregunta } \\
\text { de auditoría }\end{array}$} & \multicolumn{6}{|c|}{ Puntuación de la Evaluación } \\
\hline & & \multicolumn{3}{|c|}{ No cumple } & \multicolumn{3}{|c|}{ Si Cumple } \\
\hline 2.2 Control de las características de la calidad contable & $\begin{array}{c}9 \\
10 \\
11\end{array}$ & $\begin{array}{l}0 \\
0 \\
0\end{array}$ & $\begin{array}{l}2 \\
2 \\
2\end{array}$ & $\begin{array}{l}4 \\
4 \\
4\end{array}$ & \begin{tabular}{|l}
6 \\
6 \\
6 \\
\end{tabular} & $\begin{array}{l}8 \\
8 \\
8\end{array}$ & \begin{tabular}{|l|}
10 \\
10 \\
10 \\
\end{tabular} \\
\hline
\end{tabular}

\begin{tabular}{|c|c|c|}
\hline \multicolumn{3}{|c|}{ PUNTUACIÓN DE LA AUDITORÍA } \\
\hline $\begin{array}{c}\mathrm{N}^{\circ} \text { de referencia de } \\
\text { lineamiento de calidad }\end{array}$ & $\begin{array}{c}\text { Puntuación mínima } \\
\text { Aprobatoria }\end{array}$ & Puntuación real \\
\hline 2.2 & 18 & 22 \\
\hline
\end{tabular}

PUNTUACIÓN DE LA AUDITORÍA CONTABLE

\begin{tabular}{|c|c|c|c|c|c|}
\hline \multicolumn{6}{|c|}{ DATOS DE LA EMPRESA } \\
\hline \multicolumn{2}{|c|}{$\begin{array}{l}\text { Nombre: } \\
\text { Distrito: : }--------------------- \\
\text { Teléfono: : }------------------\end{array}$} & \multicolumn{4}{|c|}{$\begin{array}{l}\text { Dirección: : } \\
\text { Provincia: : }---------------------- \\
\text { Página web: : }----------------- \\
\end{array}$} \\
\hline \multicolumn{6}{|c|}{ CRITERIOS DE AUDITORÍA } \\
\hline \multicolumn{2}{|c|}{$\begin{array}{l}\text { Auditor: }--------------------- \\
\text { Auditado: Departamento de Contabilidad }\end{array}$} & \multicolumn{4}{|c|}{$\begin{array}{l}\text { Fecha: ........./..................... } \\
\text { Ubicación: Gerencia de Contabilidad }\end{array}$} \\
\hline $\mathbf{N}^{\circ}$ & ELEMEN & & $\begin{array}{l}\text { Puntaje } \\
\text { Aprob. } \\
\text { mínimo }\end{array}$ & $\begin{array}{c}\text { Puntaje } \\
\text { del } \\
\text { Auditor }\end{array}$ & $\begin{array}{l}\text { Puntaje } \\
\text { Aprob. } \\
\text { máximo }\end{array}$ \\
\hline 1.0 & $\begin{array}{l}\text { FUNDAMENTOS DE LA CALIDAD } \\
\text { CONTABLE. }\end{array}$ & AA A LA FUNCIÓN & & & \\
\hline 1.1 & Importancia del sistema de calidad de & contable. & 49 & 62 & 70 \\
\hline 1.2 & Características del sistema de calidad & cio contable. & 21 & 26 & 30 \\
\hline 2.0 & $\begin{array}{l}\text { CARACTERÍSTICAS DE LA CALII } \\
\text { A LA FUNCIÓN CONTABLE }\end{array}$ & RATIVA APLICADO & & & \\
\hline 2.1 & Características de la calidad contable. & & 98 & 120 & 140 \\
\hline 2.2 & Control de las características de la cal & table. & 42 & 53 & 60 \\
\hline 3.0 & PRINCIPIOS DEL SISTEMA DE CAI & ONTABLE & & & \\
\hline 3.1 & Aspectos fundamentales del sistema d & en la contabilidad. & 21 & 26 & 30 \\
\hline 3.2 & Responsabilidad ejecutiva de la contal & & 21 & 26 & 30 \\
\hline 3.3 & Desarrollo y apoyo al personal contab & & 28 & 35 & 40 \\
\hline 3.4 & Estructura del sistema de la calidad co & & 21 & 26 & 30 \\
\hline 3.4 .1 & Documentación del sistema de calida & & 21 & 26 & 30 \\
\hline 3.4 .2 & Normas del sistema de la calidad cont & & 21 & 26 & 30 \\
\hline 3.4.3 & Análisis de diferencias en el desemper & & 21 & 26 & 30 \\
\hline 3.4 .4 & Informe de auditoría de calidad del se & ntable. & 28 & 38 & 40 \\
\hline 3.4 .5 & Vigilancia de las interacciones con los & & 28 & 32 & 40 \\
\hline
\end{tabular}




\begin{tabular}{|c|l|c|c|c|}
\hline 4.0 & OPERACIONES DEL SISTEMA DE LA CALIDAD CONTABLE & & & \\
4.1 & Control del proceso contable. & 14 & 18 & 20 \\
4.1 .1 & Definición y objetivos. & 21 & $(18)$ & 30 \\
4.1 .2 & Relaciones con los clientes. & 7 & $(6)$ & 10 \\
4.1 .3 & Proyectos contables de mejora. & 14 & 18 & 20 \\
4.1 .4 & Nuevas propuestas contables. & 7 & $(6)$ & 10 \\
4.1 .5 & Registros contables normalizados y/o estandarizados. & 14 & 16 & 20 \\
4.1 .6 & Administración contable. & 21 & 26 & 30 \\
4.1 .7 & Control del proceso administrativo (P-O-C-D-C). & 7 & 8 & 10 \\
4.1 .8 & Revisión de la documentación contable. & & & \\
4.2 & Control del proceso de rediseño. & 14 & 16 & 20 \\
4.2 .1 & Historia del rediseño. & 7 & 18 & 20 \\
4.2 .2 & Identificar actividades básicas del personal contable. & 7 & 8 & 10 \\
4.2 .3 & Detectar problemas en los procesos actuales del trabajo contable. & 7 & 7 & 10 \\
4.2 .4 & Desarrollar nuevos procesos y procedimientos. & 7 & 7 & 10 \\
4.2 .5 & Desarrollar nuevas mediciones de desempeño del personal contable. & 7 & 7 & 10 \\
4.2 .6 & Especificar las nuevas normas del desempeño del personal contable. & & \\
4.2 .7 & Evaluar los nuevos procesos y procedimientos del trabajo contable. & & & \\
4.3 & Evaluación del proceso del servicio al cliente. & 14 & $(12)$ & 20 \\
4.3 .1 & Profesionalismo del personal contable en general. & 14 & $(12)$ & 20 \\
4.3 .2 & Retroalimentación del personal contable. & 14 & 16 & 20 \\
4.3 .3 & Retroalimentación de satisfacción de los clientes. & 14 & 16 & 20 \\
4.3 .4 & Control del sistema de medición. & & & \\
4.4 & Análisis de diferencias en el desempeño y la mejora contínua. & 7 & 9 & 10 \\
4.4 .1 & Ejecución y uso. & 21 & 26 & 30 \\
4.4 .2 & Sistema de recolección de datos. & 700 & 821 & 1000 \\
4.4 .3 & Iniciativa de mejora de calidad del servicio contable. \\
\hline TOTAL & PUNTAJE DE AUDITORÍA & & & \\
\hline & & & & \\
\end{tabular}

Fuente: Propia.

La auditoría de calidad al servicio contable arroja 821 puntos. Por consiguiente, el servicio está aprobado porque se encuentra en el rango de: $701-900$ puntos.

Sin embargo, para ajustar el puntaje, también podemos eliminar o sacar el puntaje negativo. Es decir, a los que tiene menos del puntaje mínimo de aprobación, o sea:

$(18)+(6)+(6)(5)+(12)+(12)+(24)=83$ puntos; daría el siguiente resultado: $821-83=738$ puntos.

NOTA: En algunos puntajes se puede apreciar que sale impar $(7,21,49$,$) , porque se le puede pre-$ sentar al auditor la disyuntiva de poner un puntaje entre el 6 y 8 , por ejemplo.

El auditor no desea poner ni 6 ni 8 entonces opta por 7 , que es un puntaje que es más justo y equitativo y que lo acerca a la verdad.

No olvidemos que a partir de aquí (el resultado obtenido), se puede y se debe fijar una política de calidad de la empresa. Con estos datos, estableceremos la base para determinar el nivel o estándar de calidad técnico que desde luego es referencial. Con la obtención de este resultado podemos obtener el nivel o estándar de política de calidad de la empresa. 


\section{CONCLUSIONES}

1. Este trabajo de investigación, puede y debe ser complementado con otro cuestionario de auditoría de calidad al servicio contable, para estudiar y analizar aquellas características que obtuvieron un puntaje menor al puntaje mínimo aprobatorio. Asimismo, nos servirá dicho cuestionario para mejorar las fortalezas de los que obtuvieron un puntaje superior al mínimo.
La puntuación de la auditoría $(62+26)$ es de 88 puntos de un máximo de 100 puntos $(70+30)$ posibles para la sección 1.0. Debido a que la norma mínima de desempeño es $(49+21) 70$ puntos y, el resultado obtenido es 88 puntos, esto significa que la performance es positiva. Aquí incluso podemos hacer una nueva tabla de puntuación que iría de menos a más.

$\begin{array}{llll}\text { Puntuación } & 70 & 88 & 100 \\ \text { Porcentaje } & 70 \% & 88 \% & 100 \%\end{array}$

\begin{tabular}{|c|c|c|c|c|}
\hline \multicolumn{5}{|c|}{ CUESTIONARIO DE AUDITORÍA DE CALIDAD } \\
\hline \multirow{3}{*}{\multicolumn{2}{|c|}{$\begin{array}{l}\text { Auditor: } \\
\text { Auditado: } \\
\text { Empresa: }\end{array}$}} & \multirow{3}{*}{\multicolumn{2}{|c|}{$\begin{array}{l}\text { Fecha: } \\
\text { Ubicación: } \\
\text { Firma del Auditor: }\end{array}$}} & \\
\hline & & & & \\
\hline & & & & \\
\hline \multirow{2}{*}{$\mathrm{N}^{\circ}$} & \multirow{2}{*}{\multicolumn{2}{|c|}{ Características de Calidad }} & \multicolumn{2}{|c|}{ Puntaje de la evaluación } \\
\hline & & & No cumple & Si cumple \\
\hline 1.0 & \multicolumn{2}{|c|}{ Fundamento de la calidad de la función o servicio contable } & & $\mathrm{X}$ \\
\hline 1.1 & \multicolumn{2}{|l|}{ Importancia de la calidad del servicio contable } & & $\mathrm{X}$ \\
\hline 1.2 & \multicolumn{2}{|c|}{ Características del sistema de calidad del servicio contable } & & $\mathrm{X}$ \\
\hline
\end{tabular}

2. Este trabajo de investigación todavía puede y debe ser complementado con una solicitud de acción correctiva.

\begin{tabular}{|c|c|c|c|c|c|}
\hline \multicolumn{6}{|c|}{ SOLICITUD DE ACCIÓN CORRECTIVA } \\
\hline \multicolumn{3}{|l|}{$\begin{array}{l}\text { Fecha: } \\
\text { Auditor: } \\
\text { Auditado: }\end{array}$} & \multicolumn{3}{|c|}{$\begin{array}{l}\text { Fecha de seguimiento de la Auditoria: } \\
\text { Firma del Auditor: } \\
\mathrm{N}^{\circ} \text { de Auditoria: }\end{array}$} \\
\hline \multirow{3}{*}{$\begin{array}{l}N^{\circ} \text { de referencia y } \\
\text { puntaje o porcen- } \\
\text { taje de prioridad }\end{array}$} & \multirow{3}{*}{$\begin{array}{l}\text { Requerimiento } \\
\text { de operaciones } \\
\text { documentadas }\end{array}$} & \multirow{3}{*}{$\begin{array}{l}\text { Observación del } \\
\text { auditor de la no } \\
\text { conformidad }\end{array}$} & \multirow{3}{*}{$\begin{array}{l}\text { Acción correctiva } \\
\text { tomada por } \\
\text { Auditado }\end{array}$} & \multicolumn{2}{|c|}{$\begin{array}{c}\text { Estado de la acción de } \\
\text { seguimiento }\end{array}$} \\
\hline & & & & \multicolumn{2}{|c|}{ De acuerdo } \\
\hline & & & & $\mathrm{Si}$ & No \\
\hline $\begin{array}{c}4.1 .2 \\
18 / 30=0.60 x \\
100 \%=60 \%\end{array}$ & $\begin{array}{l}\text { Relaciones con los } \\
\text { clientes }\end{array}$ & $\begin{array}{l}\text { Existe un manual } \\
\text { de atención al } \\
\text { cliente obsoleto. } \\
\text { No está actualizado }\end{array}$ & $\begin{array}{l}\text { Comenzó a actualizar } \\
\text { el respectivo manual } \\
\text { el día:........... }\end{array}$ & $\mathrm{x}$ & \\
\hline
\end{tabular}

3. Este método proporciona a quien lo aplique una forma práctica, sencilla e inclusive amena de auditar la calidad del servicio contable de cualquier empresa u organización.

4. Sirve para alcanzar las metas y los objetivos propuestos por el área contable.
5. Tiende al mejoramiento continuo, para alcanzar estándares de calidad mundial.

6. Se puede sacar algunas características de calidady colocar otras, a fin de ajustarlo a los requerimientos y/o necesidades de la empresa -cliente.

7. Finalmente, es el instrumento - herramienta más 
poderosa que en la actualidad tiene el Auditor de Calidad del Servicio Contable.

\section{REFERENCIAS BIBLIOGRÁFICAS}

1. George A. Smith Jr. Auditoria de calidad en la función de ventas. Panorama Editorial, S. A de C. V.,1999, México, D.F.

2. Pablo Emilio Riveros Silva. Sistema de gestión de la calidad del servicio. ECOE Ediciones, 2007, Bogotá Colombia.

3. Valdivia, R. (2011). Medición del rendimiento de la organización como sistema de control de gestión del contador. Quipukmayoc, Vol. 19 ( $\mathrm{N}^{\circ}$ 36), pp-pp.

4. Valdivia, R. (2012, julio). Un modelo de medición del rendimiento del servicio contable. Trabajo presentado en el Congreso Latinoamericano de Contabilidad y Auditoría, Machala, Ecuador.

5. Valdivia, R. (2013, octubre). Cómo medir la calidad del servicio contable. Trabajo presentado en el XVIII Congreso Internacional de Investigación en Contaduría, D.F., México. 